\title{
Molecular Serotype-Specific Identification of Non-type b Haemophilus influenzae by Loop-Mediated Isothermal Amplification
}

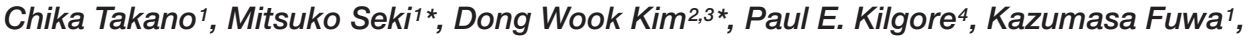
Koji Takahashi ${ }^{5}$, Toshiaki Inazaki ${ }^{6}$ and Satoshi Hayakawa'

${ }^{1}$ Division of Microbiology, Department of Pathology and Microbiology, Nihon University School of Medicine, Tokyo, Japan, ${ }^{2}$ Department of Pharmacy, College of Pharmacy, Hanyang University, Ansan, South Korea, ${ }^{3}$ Institute of Pharmacological Research, Hanyang University, Ansan, South Korea, ${ }^{4}$ Department of Pharmacy Practice, Eugene Applebaum College of Pharmacy and Health Sciences, Wayne State University, Detroit, MI, United States, ${ }^{5}$ Medical Devices Solutions Vehicle, Kaneka Corporation, Hyogo, Japan, ${ }^{6}$ Nihon University School of Medicine, Tokyo, Japan
\end{abstract}

\section{OPEN ACCESS}

Edited by:

Fumito Maruyama,

Kyoto University, Japan

Reviewed by:

Takehiko Kenzaka,

Osaka Ohtani University, Japan

Magilé De La Caridad Fonseca,

Instituto de Medicina Tropical

"Pedro Kouri", Cuba

*Correspondence:

Dong Wook Kim

dongwook@hanyang.ac.kr

Mitsuko Seki

seki.mitsuko@nihon-u.ac.jp

Specialty section:

This article was submitted to

Infectious Diseases,

a section of the journal

Frontiers in Microbiology

Received: 02 July 2017

Accepted: 13 September 2017

Published: 04 October 2017

Citation:

Takano C, Seki M, Kim DW, Kilgore PE, Fuwa K, Takahashi K, Inazaki T and Hayakawa S (2017)

Molecular Serotype-Specific Identification of Non-type b Haemophilus influenzae by Loop-Mediated Isothermal Amplification.

Front. Microbiol. 8:1877.

doi: 10.3389/fmicb.2017.01877
Over the past four decades, the incidence of meningitis caused by Haemophilus influenzae in children has decreased due to widespread vaccination against $H$. influenzae type b (Hib). The incidence of invasive diseases due to $H$. influenzae types not included in the vaccines, however, has increased. At present, there are a limited number of diagnostics available to detect non-type $\mathrm{b} \mathrm{H}$. influenzae. To address this issue, we developed a rapid, simple, and cost-effective method for detecting serotypes of H. influenzae. We designed LAMP primer sets based on published sequences for $H$. influenzae capsular types a, c, d, e, and f. The assay was evaluated to determine test reactivity, specificity, and sensitivity. To support its use in patients with suspected meningitis, we evaluated the detection limit of the non-Hib serotype specific LAMP assay using bacterial genomic DNA-spiked cerebrospinal fluid (CSF) specimens. The reactivity and specificity of the LAMP assays were confirmed using six serotypes and non-typeable $H$. influenzae strains, plus eight strains of other Haemophilus species and non-Haemophilus genera. The detection limits of the LAMP assay for capsular types a, $\mathrm{c}, \mathrm{d}$, e, and f were $10^{2}, 10^{2}, 10^{2}, 10^{3}$, and 10 copies per reaction, while those of the PCR assay were $10^{4}, 10^{4}, 10^{3}, 10^{3}$, and $10^{4}$ genome copies per reaction, respectively. Using DNA-spiked CSF specimens, the detection limit of the LAMP assay was equivalent to that using purified DNA as the template. However, the detection limit of the PCR was reduced from $10^{3}$ to $10^{4}$ genome copies per reaction for serotype $\mathrm{d}$ and from $10^{3}$ to $10^{5}$ genome copies per reaction for serotype e. To the best of our knowledge, this is the first report of a serotype-specific identification assay for $\mathrm{H}$. influenzae using the LAMP method. Our results suggest the potential of LAMP methods for patients with suspected meningitis in resource-limited laboratories or public health surveillance systems.

Keywords: loop-mediated isothermal amplification, Haemophilus influenzae, serotype identification, meningitis, cerebrospinal fluid

Abbreviations: non-Hib, Haemophilus influenzae serotypes a, c, d, e and f. 


\section{INTRODUCTION}

Haemophilus influenzae causes meningitis, epiglottitis, bacteremia, and pneumonia predominantly in infants and young children (Peltola, 2000). Over the past two decades, the introduction of $\mathrm{Hib}$ conjugate vaccines into routine immunization schedules has dramatically reduced the incidence of Hib-associated disease in many countries (Barbour, 1996). The incidence of invasive diseases due to $H$. influenzae serotypes not included in the vaccines (non-Hib strains), however, has increased (Bajanca et al., 2004; Rahman et al., 2008; Rubach et al., 2011).

Close monitoring of invasive $H$. influenzae disease continues to be important in order to understand effectiveness of $\mathrm{Hib}$ vaccines and detect emergence of invasive $H$. influenzae disease due to non-Hib strains (Ulanova and Tsang, 2014; Desai et al., 2015). To assess $H$. influenzae serotypes carriage, there is a need for laboratory facilities that can reliably cultivate $H$. influenzae and identify the capsular polysaccharide type using immunological techniques. Such facilities are found in wellequipped clinical microbiology laboratories, but serotyping of the capsular antigen can produce inconsistent results (LaClaire et al., 2003; Kim et al., 2011). Accurate H. influenzae serotyping remains a challenge due to the limited availability of serotyping methods of the capsular antigen and injudicious use of antimicrobial agents. For this reason, a reliable, non-culture based diagnostic test based on nucleic acid amplification methods has the potential to offer an important option for detection of Hib and non-Hib strains.

A PCR assay for H. influenzae serotyping (Falla et al., 1994) has been established, however, PCR-based assays are relatively expensive and complex to perform in resource-limited laboratory settings. An alternative nucleic acid detection method known as loop-mediated isothermal amplification (LAMP) utilizes a unique priming mechanism that yields specific DNA products in a shorter period of time than PCR (Notomi et al., 2000). A LAMP method for detecting Hib has been established and its clinical usefulness confirmed using clinical cerebrospinal fluid specimens (CSF) (Kim et al., 2011).

The non-Hib serotyping LAMP method has the potential to be more reliable and easier to perform than bacterial culture, antigen detection, and PCR-based assays. However, to date, a non-Hib-specific LAMP assay has not been reported. In this study, we report the establishment of a novel non-Hib serotyping LAMP assay, and compare its performance to that of conventional PCR. We also confirmed the performance of the non-Hib serotyping LAMP assay using DNA-spiked clinical CSF specimens from patients with suspected meningitis (Kennedy et al., 2007).

\section{MATERIALS AND METHODS}

\section{Bacterial Strains}

Forty-two strains of $H$. influenzae (including serotypes a to $\mathrm{f}$, non-typeable and biotype aegyptius) plus eight strains of other Haemophilus species and non-Haemophilus genera were evaluated. Additional Haemophilus species were H. parainfluenzae (HK79), H. parahaemolyticus (GTC1529), and H. haemolyticus (HK680), and non-Haemophilus genera were Streptococcus mitis (ATCC9811), S. gordonii (ATCC12396), S. pneumoniae (R6), Escherichia coli (DH5 $\alpha$ ), and Neisseria meningitidis (HY0001). In this study, 8 standard and 34 reference $H$. influenzae strains (9 Hib, and 25 non-Hib serotypes, as well as 8 non-typeable strains including 1 biotype aegyptius) were evaluated (Table 1). The eight standard $H$. influenzae strains were IID983 (serotype a), IID984 (serotype b), IID985 (serotype c), IID986 (serotype d), IID987 (serotype e), IID 988 (serotype f), IID989 (non-typeable) and IID993 (non-typeable, biotype aegyptius). The 34 reference strains included 5 serotype a (HK390, HK643, HK645, HK648, and HK649), 8 serotype b (HK176, HK177, HK179, HK180, HK195, HK196, HK827, and HK838), 4 serotype c (HK342, HK396, HK635, and HK658), 1 serotype d (HK343), 3 serotype e (HK399, HK636, and HK641), 7 serotype f (HK638, HK2055, HK2056, HK2062, HK2063, HK2065, and HK2109), and 6 non-typeable (HK856, HK2112, HK2115, HK2117, HK2119, and HK2121) strains. Capsule production by the 34 reference strains was previously confirmed by agglutination using burro antisera against serotypes a to $f$, provided by Dr. Rachel Schneerson (NIH, Bethesda, MD, United States) (Table 1).

\section{Preparation of Chromosomal DNA}

Genomic DNA was purified from the 50 strains above using a QIAamp DNA Mini Kit (QIAGEN, Valencia, CA, United States), in accordance with the manufacturer's protocol. For the detection limit study, genomic DNA from five non-Hib serotypes (IID983, IID985, IID986, IID987, and IID 988) was obtained as described above and the concentration was determined using a NanoDrop 1000 instrument (Thermo Fisher Scientific Inc., Waltham, MA, United States). The number of genome copies in the LAMP mixture was calculated based on a molecular size of $1.83 \mathrm{Mbp}(\mathrm{H}$. influenzae RD KW20; GenBank accession number, NC000907). To ascertain the detection limit of the non-Hib LAMP assay, serial tenfold dilutions of genomic DNA were amplified and the results were compared with those of conventional PCR (Falla et al., 1994).

In the detection limit study, triplicate non-Hib LAMP testing was performed over a 3-day period using serial tenfold dilutions of genomic DNA. The supernatant of pooled $H$. influenzaenegative CSF specimens (Anh et al., 2006) was used in a spiking assay, and serial tenfold dilutions of genomic non-Hib DNA were amplified by the established LAMP assay.

\section{Non-Hib LAMP Primer Design}

Five non-Hib LAMP primer sets were designed based on published sequences of the non-Hib capsulation loci (GenBank accession numbers, Z37516, HQ651151, Z33389, HM053635, and AF549211 for serotypes a, c, d, e, and f, respectively) using LAMP primer design software (FUJITSU LIMITED, 2016). The non-Hib LAMP primers included two outer primers (F3 and B3), a forward inner primer (FIP), a backward inner primer (BIP), and loop primers (LF and/or LB) (Table 2A). 
TABLE 1 | Reactivity and specificity of the non-Hib serotyping LAMP assay.

\begin{tabular}{|c|c|c|c|c|c|c|c|c|}
\hline \multirow[t]{2}{*}{ Organism } & \multirow[t]{2}{*}{ Number of strains } & \multirow[t]{2}{*}{ Origin of isolate ${ }^{a}$} & \multirow[t]{2}{*}{ Capsule type ${ }^{b}$} & \multicolumn{5}{|c|}{ Non-Hib serotyping LAMP assay } \\
\hline & & & & a & c & d & e & $\mathbf{f}$ \\
\hline \multirow[t]{7}{*}{ Standard $H$. influenzae strains $(n=8)^{d}$} & 1 & - & a & $+^{\mathrm{c}}$ & - & - & - & - \\
\hline & 1 & - & $\mathrm{b}$ & - & - & - & - & - \\
\hline & 1 & - & C & - & + & - & - & - \\
\hline & 1 & - & $d$ & - & - & + & - & - \\
\hline & 1 & - & e & - & - & - & + & - \\
\hline & 1 & - & f & - & - & - & - & + \\
\hline & 2 & - & $\mathrm{nt}$ & - & - & - & - & - \\
\hline \multirow[t]{8}{*}{ Reference $H$. influenzae strains $(n=34)^{\mathrm{e}}$} & 5 & $\mathrm{RT}$ & a & + & - & - & - & - \\
\hline & 8 & CSF & $\mathrm{b}$ & - & - & - & - & - \\
\hline & 4 & CSF & $c$ & - & + & - & - & - \\
\hline & 1 & - & $d$ & - & - & + & - & - \\
\hline & 3 & - & e & - & - & - & + & - \\
\hline & 1 & CSF & $f$ & - & - & - & - & + \\
\hline & 6 & $\mathrm{RT}$ & $f$ & - & - & - & - & + \\
\hline & 6 & RT & $\mathrm{nt}$ & - & - & - & - & - \\
\hline
\end{tabular}

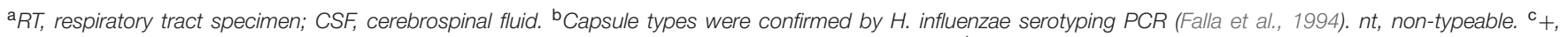

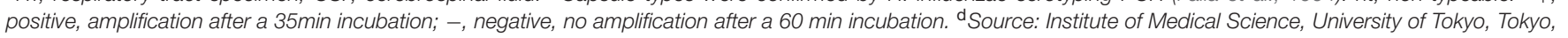
Japan. e Provided by Prof. Emeritus Mogens Kilian, Biomedicine, Aarhus University, Aarhus, Denmark.

\section{Non-Hib LAMP Reaction}

The reaction mixture $(25 \mu \mathrm{L})$ contained $1.6 \mu \mathrm{M}$ each of FIP and BIP, $0.2 \mu \mathrm{M}$ each of F3 and B3, $0.4 \mu \mathrm{M}$ of LF, $8 \mathrm{U}$ of Bst DNA polymerase large fragment (New England Biolabs, Ipswich, MA, United States), 1.4 mM deoxynucleoside triphosphates, $0.8 \mathrm{M}$ betaine (Sigma, St. Louis, MO, United States), $20 \mathrm{mM}$ Tris- $\mathrm{HCl}(\mathrm{pH} 8.8), 10 \mathrm{mM} \mathrm{KCl}, 10 \mathrm{mM}$ $\left(\mathrm{NH}_{4}\right)_{2} \mathrm{SO}_{4}, 8 \mathrm{mM} \mathrm{MgSO}_{4}, 0.1 \%$ Tween 20 , and template DNA $(2 \mu \mathrm{L})$. Each serotype reaction mixture was incubated at $63^{\circ} \mathrm{C}$ for $60 \mathrm{~min}$ and then heated at $80^{\circ} \mathrm{C}$ for $2 \mathrm{~min}$ to terminate the reaction.

\section{Analysis of Non-Hib LAMP Products}

A Loopamp real-time turbidimeter (LA-500; Eiken Chemical Co., Tokyo, Japan) was used to monitor the turbidity of the reaction tube in real-time by reading the optimal density at $650 \mathrm{~nm}$ (OD650) at 6-s intervals. The amplification time required to exceed a turbidity level of $0.1(T t)$ was calculated in accordance with the manufacturer's protocol using the turbidimeter software (Mori et al., 2004).

For the detection limit study, a colorimetric visual inspection dye (leuco triphenylmethane (Miyamoto et al., 2015); D-QUICK, Kaneka Co., Osaka, Japan) and a thermostatic color sensor (MyAbscope ${ }^{\circledR}$; Kaneka Co., Osaka, Japan; KANEKA, 2016) were used. The change in color of the reactions was observed in real time at 20-s intervals. The amplification time was determined when an absorbance level exceeded 0.1 using software of the thermostatic color sensor.

To verify their structure, the amplified LAMP products were sequenced by Akita Prefectural University Biotechnology Center using a BigDye ${ }^{\circledR}$ Terminator V3.1 cycle sequencing kit (Applied Biosystems, Foster City, CA, United States) and a 3130xL Genetic
Analyzer (Applied Biosystems). The primers used to sequence the target region are shown in Supplementary Table S1.

\section{Non-Hib PCR Assay}

Non-Hib PCR using previously reported primers (Falla et al., 1994) was performed to type the capsule of $H$. influenzae strains (Table 2B). The PCR mixture $(25 \mu \mathrm{L})$ consisted of $0.2 \mathrm{mM}$ of each deoxyribonucleoside triphosphate, $10 \mathrm{mM}$ Tris- $\mathrm{HCl}$ buffer $(\mathrm{pH}$ 8.3), $50 \mathrm{mM} \mathrm{KCl}, 2 \mathrm{mM} \mathrm{MgCl}_{2}$, $1 \mathrm{U}$ Ex Taq DNA polymerase (Takara Bio, Tokyo, Japan), $0.5 \mu \mathrm{M}$ of each primer, and $2 \mu \mathrm{L}$ of template DNA. The PCR was performed using two thermal cyclers: Veriti ${ }^{\mathrm{TM}}$ (Applied Biosystems, Foster City, CA, United States) in Japan and T-100 ${ }^{\mathrm{TM}}$ (Bio-Rad, Hercules, CA, United States) in South Korea. The reaction comprised 25 cycles, each involving $1 \mathrm{~min}$ of denaturation at $94^{\circ} \mathrm{C}, 1 \mathrm{~min}$ of annealing at $60^{\circ} \mathrm{C}$, and $1 \mathrm{~min}$ of extension at $72^{\circ} \mathrm{C}$. To detect serotype e, the reaction conditions were modified to use 35 cycles, each involving $30 \mathrm{~s}$ of denaturation at $94^{\circ} \mathrm{C}, 30 \mathrm{~s}$ of annealing at $45^{\circ} \mathrm{C}$, and $30 \mathrm{~s}$ of extension at $72^{\circ} \mathrm{C}$ (Satola et al., 2007). Following the final cycle, all reactions were incubated for further $10 \mathrm{~min}$ at $72^{\circ} \mathrm{C}$. Products were visualized by resolution in an agarose gel followed by staining with ethidium bromide.

\section{DNA Spiked Clinical CSF Specimens}

To conduct a pilot evaluation of the non-Hib LAMP, seven bexA PCR-negative specimens were randomly selected from CSF collected during a 2-year prospective study of bacterial meningitis in Hanoi (Anh et al., 2006). CSF specimens were pre-treated at $95^{\circ} \mathrm{C}$ for $2 \mathrm{~min}$ and centrifuged $(13,000 \times \mathrm{g}, 5 \mathrm{~min})$. The supernatant of the seven clinical CSF specimens was preserved for use in DNA-spiked CSF experiments. A $2 \mu \mathrm{L}$ aliquot of DNAspiked CSF specimen was subjected to non-Hib serotyping PCR 
TABLE 2 | (A) LAMP Primer sequences in this study.

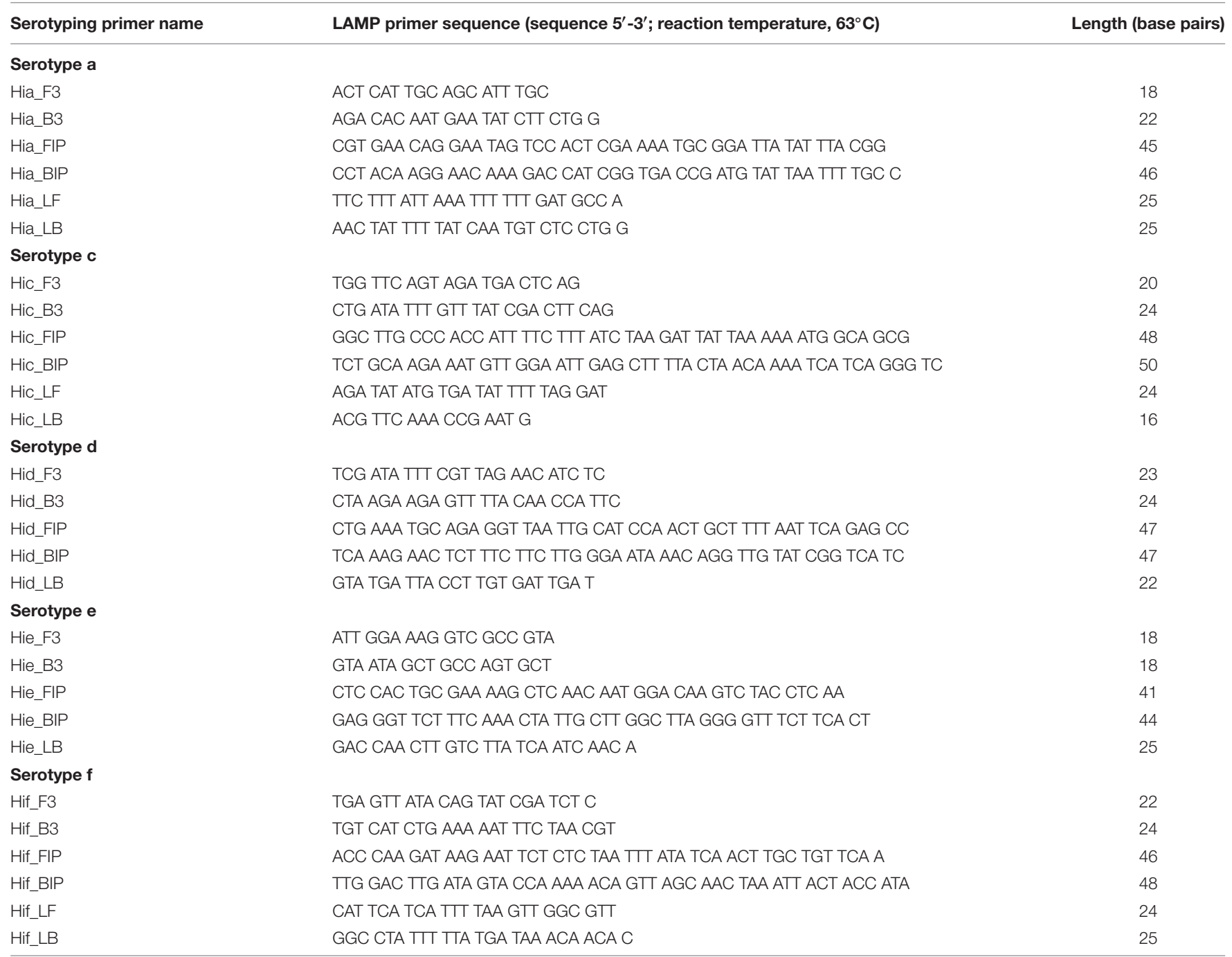

TABLE 2 | (B) PCR Primer sequences in this study (Falla et al., 1994).

\section{Serotyping primer name}

\section{Serotype a}

a1

a2

\section{Serotype c}

c1

c2

\section{Serotype d}

d1

d2

\section{Serotype e}

e1

e2

\section{Serotype f}

f1

f2
PCR primer sequence (sequence $5^{\prime}-3^{\prime}$ )

Length (base pairs)

CTA CTC ATT GCA GCA TाT GC

GAA TAT GAC CTG ATC TTC TG

20

20

TCT GTG TAG ATG ATG GTT CA

CAG AGG CAA GCT ATT AGT GA

20

20

TGA TGA CCG ATA CAA CCT GT

20

TCC ACT CTT CAA ACC ATT CT

20

GGT AAC GAA TGT AGT GGT AG

20

GCT TTA CTG TAT AAG TCT AG

20

GCT ACT ATC AAG TCC AAA TC

20

CGC AAT TAT GGA AGA AAG CT 


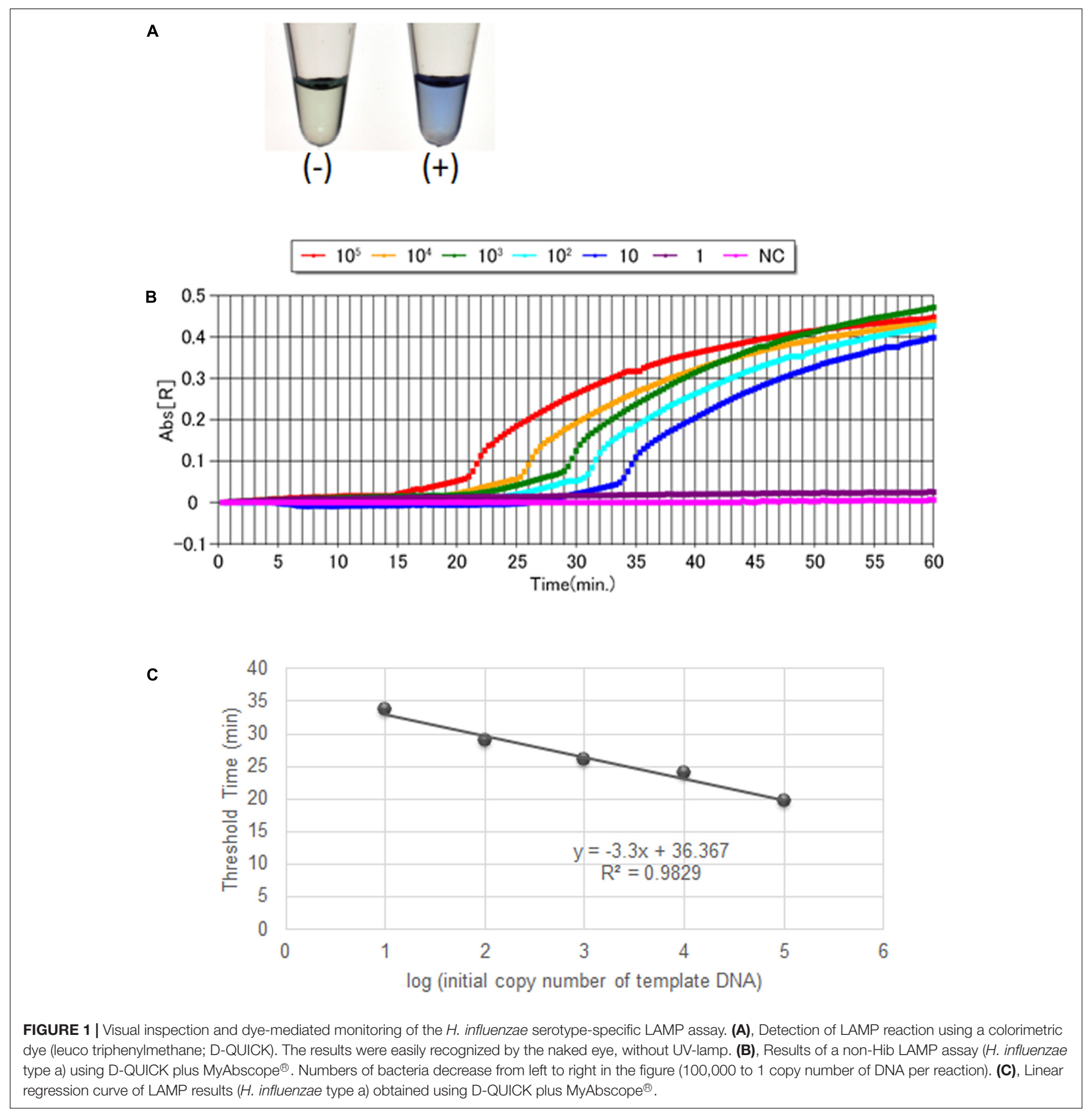

and non-Hib serotyping LAMP as described above at Hanyang University, South Korea.

\section{Ethics Statement}

We utilized CSF specimens preserved from our previous surveillance study (Anh et al., 2006; Kennedy et al., 2007). All CSF specimens utilized in this study were de-identified prior to laboratory processing and analysis. Ethical approval for patient specimen collection during surveillance was obtained from the following ethics review committees: the Institutional Review
Board of the International Vaccine Institute, Seoul, South Korea; and the Institutional Review Board at the National Institute of Hygiene and Epidemiology, Hanoi, Vietnam. Each institution participated in prospective, population-based surveillance for childhood meningitis from 1999 to 2002 (Anh et al., 2006; Kennedy et al., 2007). During those surveillance studies, written consent was not obtained as the CSF collection was considered routine standard care for hospitalized children with suspected bacterial meningitis. For this reason, verbal consent of the parent or legal guardian present with the child during the period of 
hospitalization was recorded in the patient's medical chart at the time of the clinical lumbar puncture procedure. This consent procedure was approved by these local scientific ethics review committees of the participating institutions.

\section{RESULTS}

The non-Hib LAMP assay for capsular types a, c, d, e, and $\mathrm{f}$ successfully amplified each target sequence of non-Hib capsulation loci (Table 1). The products were confirmed by visual inspection of turbidity/color of the reaction tube, real-time turbidimetry and real-time colorimetric sensor (Figure 1).

\section{Analytical Reactivity and Specificity of the Non-Hib LAMP}

To evaluate the analytical reactivity and specificity of the nonHib LAMP primers, we tested $42 \mathrm{H}$. influenzae strains and 8 non- $H$. influenzae strains. For each assay mixture, a standard genomic DNA concentration $\left(10^{5}\right.$ copies $)$ was used for each strain. The non-Hib LAMP reaction for capsular types a, c, d, e, and $f$ amplified each non-Hib target DNA sequence regardless of the different sources (i.e., respiratory tract or cerebrospinal fluid specimens). In contrast, genomic DNA of Hib or other target capsular types was not amplified. Neither non-typeable $H$. influenzae nor the eight standard non- $H$. influenzae species yielded a positive result in the non-Hib LAMP reaction (Table 1).

Amplification specificity was confirmed by sequencing, and the sequences were compared with those of the target region in the original sequence of the non-Hib capsulation locus (between F1 and B1; Supplementary Figure S1). The sequences obtained were identical to the expected nucleotide sequences (data not shown).

\section{Detection Limit of the Non-Hib LAMP Reaction}

The detection limits of the LAMP assays for capsular types a, c, $\mathrm{d}$, e, and $\mathrm{f}$ were $10^{2}, 10^{2}, 10^{2}, 10^{3}$, and 10 copies per reaction, while those of the PCR assays were $10^{4}, 10^{4}, 10^{3}, 10^{3}$, and $10^{4}$ genome copies per reaction, respectively. Thus, the sensitivity of the non-Hib LAMP assay was 1-1,000-fold greater than that of non-Hib PCR (Table 3). The detection limits of each serotype LAMP assay were identical between the real-time measurement and direct visual inspection. No amplification was apparent in the non-Hib LAMP reaction for samples lacking target DNA. The above results were obtained in triplicate over 3 days and identical results were obtained in the laboratories in Japan and South Korea.

The results of LAMP assays using D-QUICK plus MyAbscope $^{\circledR}$ are shown in Figure 1. The color change of the LAMP products was more easily recognized than an increase in turbidity. The real-time measurement results were used to generate a linear regression curve with high linearity, while the observation period was longer than that of real-time turbidimetry (20-s vs. 6-s intervals) (Figure 1C). The total reaction time of the two methods was the same $(60 \mathrm{~min})$.

\section{LAMP Analysis of DNA-Spiked CSF Specimens}

The detection limit of the non-Hib LAMP assay using DNA spiked CSF specimens was 10-1,000 genome copies, identical to that using purified DNA as the template (Table 3). However, that of the non-Hib PCR worsened from $10^{3}$ to $10^{4}$ genome copies per reaction for serotype $\mathrm{d}$ and from $10^{3}$ to $10^{5}$ genome copies per reaction for serotype e. The detection limit of the non-Hib LAMP assay was identical between direct visual inspection and real-time measurement.

\section{DISCUSSION}

This is the first report of a serotype-specific identification assay for $H$. influenzae using the LAMP method. The non-Hib LAMP assay established in this study accurately identified various standard and reference non-Hib strains. The non-Hib LAMP demonstrated an analytical specificity equivalent to that of nonHib PCR. Notably, the non-Hib LAMP assay was found to have a detection limit 1-1,000-fold more sensitive than previously described non-Hib PCR methods (Falla et al., 1994; Corless et al., 2001). Although we used spiked CSF samples, the nonHib LAMP reaction exhibited sensitivity equivalent to that when purified DNA was used as the template. However, the detection limit of the non-Hib PCR for serotypes $d$ and e was reduced when spiked CSF samples were used. The superior detection limit and high robustness (Francois et al., 2011) of the LAMP assay may explain its superior detection limit to that of non-Hib PCR.

In children under 5 years of age, incidence of invasive non-Hib diseases has increased globally since the widespread introduction of Hib vaccines. Notably, in adults 65 years of age and older, the number of clinical infections with non-Hib strains have also increased recently (Desai et al., 2015). These studies suggest that the most common and important non-Hib strain are nontypeable, and encapsulated non-Hib strains (serotypes a, c, d, $\mathrm{e}$, and $\mathrm{f}$ ) began to be reported after the introduction of Hib vaccination (Dworkin et al., 2007; Resman et al., 2011; Ulanova and Tsang, 2014). Thus, the incidence of invasive non-Hib

TABLE 3 | Detection limits of LAMP and PCR assays detecting DNAs of non-Hib serotypes and using the DNA spiked CSF specimens.

\begin{tabular}{|c|c|c|c|c|}
\hline \multirow[t]{2}{*}{$\begin{array}{l}\text { Non-Hib } \\
\text { serotypes }\end{array}$} & \multicolumn{2}{|c|}{$\begin{array}{l}\text { Detection limit } \\
\text { (Purified DNA) }\end{array}$} & \multicolumn{2}{|c|}{$\begin{array}{l}\text { Detection limit } \\
\text { (Spiked CSF) }\end{array}$} \\
\hline & $\mathrm{PCR}^{\mathrm{a}, \mathrm{b}}$ & LAMPa $^{a}$ & $\mathrm{PCR}^{\mathrm{a}, \mathrm{b}}$ & LAMPa $^{a}$ \\
\hline $\mathrm{a}$ & $10^{4}$ copies $^{\mathrm{C}}$ & $10^{2}$ & $10^{4}$ copies $^{\mathrm{c}}$ & $10^{2}$ \\
\hline c & $10^{4}$ & $10^{2}$ & $10^{4}$ & $10^{2}$ \\
\hline$d$ & $10^{3}$ & $10^{2}$ & $10^{4}$ & $10^{2}$ \\
\hline e & $10^{3}$ & $10^{3}$ & $10^{5}$ & $10^{3}$ \\
\hline$f$ & $10^{4}$ & 10 & $10^{4}$ & 10 \\
\hline
\end{tabular}

aPCR results were obtained by electrophoretic analysis. LAMP results were determined visually. ' ${ }^{b}$ Conventional PCR (Falla et al., 1994). ' Amount of DNA per reaction. 
disease should be monitored and its clinical and epidemiological characteristics should be thoroughly investigated.

Existing assays for non-Hib infections have notable limitations. Conventional slide agglutination serotyping tests can produce misidentifications (LaClaire et al., 2003). However, in our study, the non-Hib LAMP assay successfully distinguished each non-Hib serotype strain. The non-Hib LAMP assay used in this study was analytically specific and had a superior detection limit to conventional PCR serotyping. Our previous reports of LAMP assays to detect $H$. influenzae type b, S. pneumoniae and $N$. meningitidis also revealed high sensitivity of the assays. The detection limits were 10-102 DNA copies per reaction. The high sensitivity of the novel non-Hib LAMP assay is consistent with the previous studies (Kim et al., 2011, 2012; Lee et al., 2015a,b).

As the LAMP reaction progresses, the by-product pyrophosphate ions bind to magnesium ions and form a white precipitate of magnesium pyrophosphate. The resulting turbidity can be visualized by the naked eye. This characteristic feature of the LAMP reaction can be used to detect the reaction end-point, by identifying the presence of precipitate. The Loopamp real-time turbidimeter enables quantitative analysis of minute amounts of nucleic acids (Mori et al., 2004).

In the present study, we assessed the amount of non-Hib serotype template DNA in real-time using the D-QUICK plus MyAbscope $^{\circledast}$. To our knowledge, this is the first report of a LAMP assay using D-QUICK plus MyAbscope ${ }^{\circledR}$ to quantify DNA. D-QUICK uses leuco triphenylmethane dye, which binds to double stranded DNA; a positive reaction is indicated by a change from colorless to violet (Miyamoto et al., 2015). This involves direct colorimetric detection of LAMP amplified products, rather than a by-product of the LAMP reaction, and the instrument measures the absorbance of the reaction in real time at 20-s intervals (KANEKA, 2016). As shown in Figure 1C, the real-time measurement had the linear regression curve with high linearity. Moreover, the simpler real-time absorbance measurement is less costly than the real-time turbidity measurement.

Previous studies demonstrated that the LAMP reaction is more tolerant of the presence of potentially perturbing biological substances (i.e., reaction inhibitors) than PCR (Kaneko et al., 2007). Therefore, LAMP assays may be suitable in resourcelimited settings in developed and developing countries. The robust performance of the non-Hib LAMP assay (Francois et al., 2011) in the present study also suggests that LAMP-based detection of non-Hib and other invasive bacterial pathogens is feasible in a wide variety of clinical settings such as hospitals and health system clinical laboratories. In addition, compared with non-Hib PCR assays, the non-Hib LAMP assay has greater analytical sensitivity and less likely to miss clinical disease caused by non-Hib infections.

\section{REFERENCES}

Anh, D. D., Kilgore, P. E., Kennedy, W. A., Nyambat, B., Long, H. T., Jodar, L., et al. (2006). Haemophilus influenzae type B meningitis among children in Hanoi, Vietnam: epidemiologic patterns and estimates of $H$. Influenzae type B disease burden. Am. J. Trop. Med. Hyg. 74, 509-515.
Our laboratory's experience with the non-Hib LAMP and LAMP for the detection of other pathogens, such as S. pneumoniae, suggests that the cost of the non-Hib LAMP assay per specimen tested is markedly lower than that of PCR, and can be performed in laboratories with limited technology. Prospective studies of the non-Hib LAMP assay using clinical specimens that are now underway will confirm the assay's sensitivity, specificity, predictive values, and likelihood ratios compared with bacterial culture, antigen detection, and PCR.

In summary, we have successfully established a LAMP-based non-Hib DNA amplification method and confirmed its superior analytical specificity and detection limit. Compared with PCRbased detection methods, this assay enables the detection of nonHib serotypes with high sensitivity. Because the LAMP reaction is easy to set-up and does not require specialized equipment, it has obvious advantages in clinical settings and in population-based studies with limited access to well-equipped laboratories.

\section{AUTHOR CONTRIBUTIONS}

PK, DK, MS, and SH contributed the conception of this study; KT, DK, and MS designed the experiments; CT, KF, TI, DK, MS, and KT performed the experiments; PK acquired samples; CT, KT, DK, and MS analyzed data; CT, SH, PK, DK, and MS interpreted data; CT, PK, SH, DK, and MS drafted the manuscript; and KF, $\mathrm{TI}$, and KT approved the manuscript.

\section{FUNDING}

This project was supported by JSPS Bilateral Open Partnership Joint Research Projects (MS). DK was supported by the grant NRF-2015R1A2A2A01007297 and NRF2015M3C9A2054024 from National Research Foundation (NRF) of Korea.

\section{ACKNOWLEDGMENTS}

We thank all staff of the Department of Pharmacy, College of Pharmacy, Hanyang University. We are grateful to Prof. Morgens Kilian (Aarhus University) for his support in this study.

\section{SUPPLEMENTARY MATERIAL}

The Supplementary Material for this article can be found online at: http://journal.frontiersin.org/article/10.3389/fmicb. 2017.01877/full\#supplementary-material

Bajanca, P., Canica, M., and Multicenter Study, G. (2004). Emergence of nonencapsulated and encapsulated non-b-type invasive Haemophilus influenzae isolates in Portugal (1989-2001). J. Clin. Microbiol. 42, 807-810. doi: 10.1128/JCM.42.2.807-810.2004

Barbour, M. L. (1996). Conjugate vaccines and the carriage of Haemophilus influenzae type b. Emerg. Infect. Dis. 2, 176-182. doi: 10.3201/eid0203.960303 
Corless, C. E., Guiver, M., Borrow, R., Edwards-Jones, V., Fox, A. J., and Kaczmarski, E. B. (2001). Simultaneous detection of Neisseria meningitidis, Haemophilus influenzae, and Streptococcus pneumoniae in suspected cases of meningitis and septicemia using real-time PCR. J. Clin. Microbiol. 39, 1553-1558. doi: 10.1128/JCM.39.4.1553-1558.2001

Desai, S., Jamieson, F. B., Patel, S. N., Seo, C. Y., Dang, V., Fediurek, J., et al. (2015). The epidemiology of invasive Haemophilus influenzae non-serotype B disease in Ontario, Canada from 2004 to 2013. PLOS ONE 10:e0142179. doi: 10.1371/journal.pone.0142179

Dworkin, M. S., Park, L., and Borchardt, S. M. (2007). The changing epidemiology of invasive Haemophilus influenzae disease, especially in persons $\geq 65$ years old. Clin. Infect. Dis. 44, 810-816. doi: 10.1086/511861

Falla, T. J., Crook, D. W., Brophy, L. N., Maskell, D., Kroll, J. S., and Moxon, E. R. (1994). PCR for capsular typing of Haemophilus influenzae. J. Clin. Microbiol. 32, 2382-2386.

Francois, P., Tangomo, M., Hibbs, J., Bonetti, E. J., Boehme, C. C., Notomi, T., et al. (2011). Robustness of a loop-mediated isothermal amplification reaction for diagnostic applications. FEMS Immunol. Med. Microbiol. 62, 41-48. doi: 10.1111/j.1574-695X.2011.00785.x

FUJITSU LIMITED (2016). PrimerExplorer V5. Available at: http://primerexplorer. jp/e/ [accessed 2016].

KANEKA (2016). Thermostatic Color Sensor MyAbscope. Available at: https://www. kaneka-labtest.com/en/instrument/index.html [accessed 2016].

Kaneko, H., Kawana, T., Fukushima, E., and Suzutani, T. (2007). Tolerance of loop-mediated isothermal amplification to a culture medium and biological substances. J. Biochem. Biophys. Methods 70, 499-501. doi: 10.1016/j.jbbm.2006. 08.008

Kennedy, W. A., Chang, S. J., Purdy, K., Le, T., Kilgore, P. E., Kim, J. S., et al. (2007). Incidence of bacterial meningitis in Asia using enhanced CSF testing: polymerase chain reaction, latex agglutination and culture. Epidemiol. Infect. 135, 1217-1226. doi: 10.1017/S0950268806007734

Kim, D. W., Kilgore, P. E., Kim, E. J., Kim, S. A., Anh, D. D., Dong, B. Q., et al. (2012). The enhanced pneumococcal LAMP assay: a clinical tool for the diagnosis of meningitis due to Streptococcus pneumoniae. PLOS ONE 7:e42954. doi: 10.1371/journal.pone.0042954

Kim, D. W., Kilgore, P. E., Kim, E. J., Kim, S. A., Anh, D. D., and Seki, M. (2011). Loop-mediated isothermal amplification assay to detect Haemophilus influenzae type b in cerebrospinal fluid. J. Clin. Microbiol. 49, 3621-3626. doi: 10.1128/JCM.0051511

LaClaire, L. L., Tondella, M. L., Beall, D. S., Noble, C. A., Raghunathan, P. L., Rosenstein, N. E., et al. (2003). Identification of Haemophilus influenzae serotypes by standard slide agglutination serotyping and PCR-based capsule typing. J. Clin. Microbiol. 41, 393-396. doi: 10.1128/JCM.41.1.393-396.2003

Lee, D., Kim, E. J., Kilgore, P. E., Kim, S. A., Takahashi, H., Ohnishi, M., et al. (2015a). Clinical evaluation of a loop-mediated isothermal amplification (LAMP) assay for rapid detection of Neisseria meningitidis in cerebrospinal fluid. PLOS ONE 10:e0122922. doi: 10.1371/journal.pone.0122922

Lee, D., Kim, E. J., Kilgore, P. E., Takahashi, H., Ohnishi, M., Tomono, J., et al. (2015b). A novel loop-mediated isothermal amplification assay for serogroup identification of Neisseria meningitidis in cerebrospinal fluid. Front. Microbiol. 6:1548. doi: 10.3389/fmicb.2015.01548
Miyamoto, S., Sano, S., Takahashi, K., and Jikihara, T. (2015). Method for colorimetric detection of double-stranded nucleic acid using leuco triphenylmethane dyes. Anal. Biochem. 473, 28-33. doi: 10.1016/j.ab.2014. 12.016

Mori, Y., Kitao, M., Tomita, N., and Notomi, T. (2004). Real-time turbidimetry of LAMP reaction for quantifying template DNA. J. Biochem. Biophys. Methods 59, 145-157. doi: 10.1016/j.jbbm.2003.12.005

Notomi, T., Okayama, H., Masubuchi, H., Yonekawa, T., Watanabe, K., Amino, N., et al. (2000). Loop-mediated isothermal amplification of DNA. Nucleic Acids Res. 28, E63. doi: 10.1093/nar/28.12.e63

Peltola, H. (2000). Worldwide Haemophilus influenzae type b disease at the beginning of the 21st century: global analysis of the disease burden 25 years after the use of the polysaccharide vaccine and a decade after the advent of conjugates. Clin. Microbiol. Rev. 13, 302-317. doi: 10.1128/CMR.13.2.302-317. 2000

Rahman, M., Hossain, S., Baqui, A. H., Shoma, S., Rashid, H., Nahar, N., et al. (2008). Haemophilus influenzae type-b and non-b-type invasive diseases in urban children (<5years) of Bangladesh: implications for therapy and vaccination. J. Infect. 56, 191-196. doi: 10.1016/j.jinf.2007. 12.008

Resman, F., Ristovski, M., Ahl, J., Forsgren, A., Gilsdorf, J. R., Jasir, A., et al. (2011). Invasive disease caused by Haemophilus influenzae in Sweden 19972009; evidence of increasing incidence and clinical burden of non-type b strains. Clin. Microbiol. Infect. 17, 1638-1645. doi: 10.1111/j.1469-0691.2010. 03417.x

Rubach, M. P., Bender, J. M., Mottice, S., Hanson, K., Weng, H. Y., Korgenski, K., et al. (2011). Increasing incidence of invasive Haemophilus influenzae disease in adults, Utah, USA. Emerg. Infect. Dis. 17, 1645-1650. doi: 10.3201/eid1709. 101991

Satola, S. W., Collins, J. T., Napier, R., and Farley, M. M. (2007). Capsule gene analysis of invasive Haemophilus influenzae: accuracy of serotyping and prevalence of IS1016 among nontypeable isolates. J. Clin. Microbiol. 45, 3230-3238. doi: 10.1128/JCM.00794-07

Ulanova, M., and Tsang, R. S. (2014). Haemophilus influenzae serotype a as ause of serious invasive infections. Lancet Infect. Dis. 14, 70-82. doi: 10.1016/S14733099(13)70170-1

Conflict of Interest Statement: $\mathrm{SH}$ has received research grant funding from Kaneka Co., Ltd. KT is an employee of Kaneka Co., Ltd.

The other authors declare that the research was conducted in the absence of any commercial or financial relationships that could be construed as a potential conflict of interest.

Copyright (c) 2017 Takano, Seki, Kim, Kilgore, Fuwa, Takahashi, Inazaki and Hayakawa. This is an open-access article distributed under the terms of the Creative Commons Attribution License (CC BY). The use, distribution or reproduction in other forums is permitted, provided the original author(s) or licensor are credited and that the original publication in this journal is cited, in accordance with accepted academic practice. No use, distribution or reproduction is permitted which does not comply with these terms. 